\title{
La movilidad geográfica de búlgaros y rumanos durante la Gran Recesión en España
}

\author{
Rafael Viruela \\ Universitat de València. Departament de Geografia \\ raviruel@uv.es
}

Recepción: julio de 2014

Aceptación: enero de 2015

\begin{abstract}
Resumen
El objetivo de este artículo es analizar las pautas de movilidad de los inmigrantes procedentes de Bulgaria y Rumania durante la grave crisis económico-laboral que afecta a España. La información utilizada procede de entrevistas en profundidad a migrantes búlgaros y rumanos, que se completa con datos del Instituto Nacional de Estadística y del Ministerio de Empleo y Seguridad Social. Los resultados muestran el fuerte incremento de la movilidad interprovincial de trabajadores, sobre todo en los primeros años y con un destacado protagonismo de los varones, y el progresivo aumento de la migración internacional, que adopta diversas formas: retorno, migración circular entre el lugar de origen y el de destino y emigración a un tercer país. Han emigrado familias completas, pero los hombres también predominan en las salidas.
\end{abstract}

Palabras clave: España; inmigrantes búlgaros y rumanos; crisis económica; movilidad geográfica.

\section{Resum. La mobilitat geogràfica de búlgars i romanesos durant la Gran Recessió a Espanya}

L'objectiu d'aquest article és analitzar les pautes de mobilitat dels immigrants procedents de Bulgària i Romania durant la greu crisi econòmica i laboral que afecta l'Estat espanyol. La informació utilitzada procedeix d'entrevistes en profunditat a migrants búlgars i romanesos, que es completa amb dades de l'Institut Nacional d'Estadística i del Ministeri de Treball i Seguretat Social. Els resultats mostren el notable augment de la mobilitat interprovincial dels treballadors, sobretot en els primers anys i amb un destacat protagonisme dels homes, i el progressiu augment de la migració internacional, que adopta diverses formes: retorn, migració circular entre el lloc d'origen i el de destinació i emigració a un tercer país. Han emigrat famílies completes, però els homes també predominen en les sortides.

Paraules clau: Espanya; immigrants búlgars i romanesos; crisi econòmica; mobilitat geogràfica. 
Résumé. Mobilité géographique des Bulgares et Roumains pendant la Grande Récession en Espagne

L'objectif de cet article est d'analyser les schémas de mobilité des immigrants en provenance de la Bulgarie et de la Roumanie au cours de la crise économique et du travail qui affecte l'Espagne. L'information employée provient d'entretiens avec des migrants bulgares et roumains, ainsi que des données de l'Institut National de la Statistique et du Ministère du Travail et des Affaires Sociales. Les résultats indiquent une augmentation considérable de la mobilité interprovinciale des travailleurs, en particulier dans les premières années et avec un rôle prédominant des hommes, et un accroissement progressif de la migration internationale qui prend des formes diverses : retour, migration circulaire entre le lieu d'origine et de destination, et émigration vers un troisième pays. Des familles entières ont émigré, mais les hommes dominent aussi les sorties.

Mots-clés: Espagne; immigrés bulgares et roumains; crise économique; mobilité géographique.

Abstract. The geographic mobility of Bulgarians and Romanians during the Great Recession in Spain

The aim of this paper is to analyze the mobility patterns of immigrants coming from Bulgaria and Romania during the severe economic and employment crisis affecting Spain. The information was drawn from interviews of Bulgarian and Romanian migrants and supplemented with data from the National Institute of Statistics and the Ministry of Labor and Social Security. The results show a strong increase in the interprovincial mobility of workers, especially for men in the early years of the crisis, and the progressive increase in international migration, which takes various forms: return, circular migration between home and host areas, and emigration to a third country. Whole families have emigrated, but males still dominate emigration.

Keywords: Spain; Romanian and Bulgarian immigration; economic crisis; geographic mobility.

\section{Sumario}

1. Introducción 5. Estrategias de movilidad internacional

2. Objetivo: el estudio de la movilidad en tiempos de crisis

6. Conclusión

3. Metodología

Referencias bibliográficas

4. La movilidad geográfica interior 


\section{Introducción}

España ha recibido un gran número de inmigrantes en las dos últimas décadas, sobre todo en los primeros años del siglo XxI: de 1,5 millones de residentes nacidos en el extranjero en enero de 2000 ha pasado a contar con 6,6 millones en el momento actual ( 1 de enero de 2013). La mayor parte de los inmigrantes proceden de países con menores niveles de renta, atraídos por la impresionante oferta de empleo que ha generado un crecimiento económico excepcional, impulsado por actividades muy intensivas en mano de obra poco cualificada, como la construcción, el servicio doméstico, las actividades relacionadas con el turismo o la agricultura de regadío (Torres, 2011). Los recién llegados han ocupado más de la mitad de los más de cinco millones de empleos netos que se crearon entre 2000 y 2008 (Pajares, 2010). Al peculiar modelo de desarrollo español hay que añadir la reducida oferta de trabajadores autóctonos, como consecuencia de la rápida reducción de la fecundidad a partir de mediados de los años 1970 y del abandono de los sectores de actividad que por sus condiciones de trabajo los españoles no consideran aceptables (Cachón, 2009; Moreno y Bruquetas, 2011).

Buena parte de los migrantes proceden de Europa del Este (1,3 millones), con un destacado protagonismo de los oriundos de Rumania y Bulgaria, que empezaron a llegar a España a mediados de los años noventa. Al principio España atrajo a unos pocos (en enero de 2000, había 3.200 inmigrantes nacidos en Bulgaria y 7.500 en Rumania), pero en un breve periodo de tiempo se ha convertido en uno de los destinos más importantes para ambos colectivos (OECD, 2007; World Bank, 2010; European Commission, 2011), sobre todo para los rumanos que, con más de 800.000 efectivos en 2013, ocupan la primera posición entre la población nacida en el extranjero (el 12\% del total). Por su parte, la comunidad búlgara en España cuenta con 160.000 residentes (Tabla 1).

En el rápido y espectacular aumento de la inmigración procedente de Europa del Este confluyen varios factores: las dificultades para establecerse en otros países de su preferencia, la amplia oferta de empleo en España y las diferencias salariales con respecto a los países de origen, o el rápido fortalecimiento de las cadenas y redes migratorias construidas sobre vínculos familiares o de proximidad geográfica o social (Şerban y Voicu, 2010; Gómez y Molina, 2010; Viruela, 2011). Las decisiones político-administrativas han tenido una gran incidencia en esta corriente migratoria: la exención de visado (mayo de 2001, en el caso de los búlgaros, enero de 2002, en el de los rumanos), los acuerdos en materia de inmigración y, sobre todo, la incorporación de Rumania y Bulgaria a la Unión Europea en 2007, que conforma un espacio de libre circulación.

Las comunidades rumana y búlgara que residen en España tienen características sociodemográficas similares. Son poblaciones jóvenes, con elevada representación de los grupos de edad activa (más de la mitad tienen entre 25 y 45 años), como corresponde a una inmigración laboral cuya tasa de actividad (alrededor del 85\% en 2011) es de las más altas entre la población inmigrada 
Tabla 1. Evolución de la población nacida en Bulgaria y Rumania y residente en España (1998-2014)

\begin{tabular}{|c|c|c|c|c|c|c|}
\hline \multirow[b]{2}{*}{ Año } & \multicolumn{3}{|c|}{ Bulgaria } & \multicolumn{3}{|c|}{ Rumania } \\
\hline & Stock & $\begin{array}{c}\% \text { sobre } \\
\text { el total de } \\
\text { extranjeros }\end{array}$ & $\begin{array}{c}\text { Variación } \\
\text { anual }\end{array}$ & Stock & $\begin{array}{c}\% \text { sobre } \\
\text { el total de } \\
\text { extranjeros }\end{array}$ & $\begin{array}{c}\text { Variación } \\
\text { anual }\end{array}$ \\
\hline 1998 & 1.550 & 0,1 & & 3.066 & 0,3 & \\
\hline 1999 & 2.025 & 0,2 & 475 & 4.038 & 0,3 & 972 \\
\hline 2000 & 3.268 & 0,2 & 1.243 & 7.543 & 0,5 & 3.505 \\
\hline 2001 & 12.413 & 0,6 & 9.145 & 33.044 & 1,7 & 25.501 \\
\hline 2002 & 30.210 & 1,2 & 17.797 & 68.561 & 2,6 & 35.517 \\
\hline 2003 & 53.391 & 1,6 & 23.181 & 137.834 & 4,2 & 69.273 \\
\hline 2004 & 70.364 & 1,9 & 16.973 & 206.395 & 5,6 & 68.561 \\
\hline 2005 & 92.971 & 2,1 & 22.607 & 312.099 & 7,1 & 105.704 \\
\hline 2006 & 100.763 & 2,1 & 7.792 & 397.270 & 8,2 & 85.171 \\
\hline 2007 & 120.151 & 2,3 & 19.388 & 510.983 & 9,7 & 113.713 \\
\hline 2008 & 150.742 & 2,5 & 30.591 & 706.164 & 11,7 & 195.181 \\
\hline 2009 & 159.993 & 2,5 & 9.251 & 762.163 & 11,8 & 55.999 \\
\hline 2010 & 163.550 & 2,5 & 3.557 & 784.834 & 11,9 & 22.671 \\
\hline 2011 & 165.668 & 2,5 & 2.118 & 810.348 & 12,1 & 25.514 \\
\hline 2012 & 168.124 & 2,5 & 2.456 & 833.764 & 12,3 & 23.416 \\
\hline 2013 & 160.157 & 2,4 & 7.967 & 801.412 & 12,1 & 32.352 \\
\hline $2014^{\star}$ & 142.248 & 2,8 & 17.909 & 725.137 & 14,5 & 76.275 \\
\hline
\end{tabular}

Fuente: INE, Padrón de Habitantes, datos a 1 de enero de cada año. Elaboración propia.

*Datos provisionales en 2014.

(Colectivo Ioé, 2012). En ambos grupos destaca el relativo equilibrio entre sexos (las mujeres suponen el $48 \%$ de los búlgaros y el $49 \%$ de los rumanos), característico de una inmigración familiar. Durante la fase de crecimiento económico y del empleo, encontraron trabajo con facilidad y era habitual que trabajaran todos los miembros de la familia en edad activa. La construcción, en el caso de los hombres, y el servicio doméstico, en el de las mujeres, han funcionado como la puerta de entrada al mercado laboral, con una destacada presencia de unos y otras en la agricultura (Colectivo Ioé y Fernández, 2010).

El volumen alcanzado por ambos colectivos ha estado acompañado de un rápido proceso de difusión espacial que se ha producido en muy poco tiempo y ha generalizado la presencia de búlgaros y rumanos en todas las regiones españolas (Figura 1). No obstante, la distribución geográfica se caracteriza por la mayor concentración en Madrid y en la Comunidad Valenciana, que reúnen el 40\% del total, además de Castilla y León, en el caso búlgaro, en relación con las oportunidades laborales y de vivienda y con la presencia de redes sociales de apoyo (Viruela 2008). 


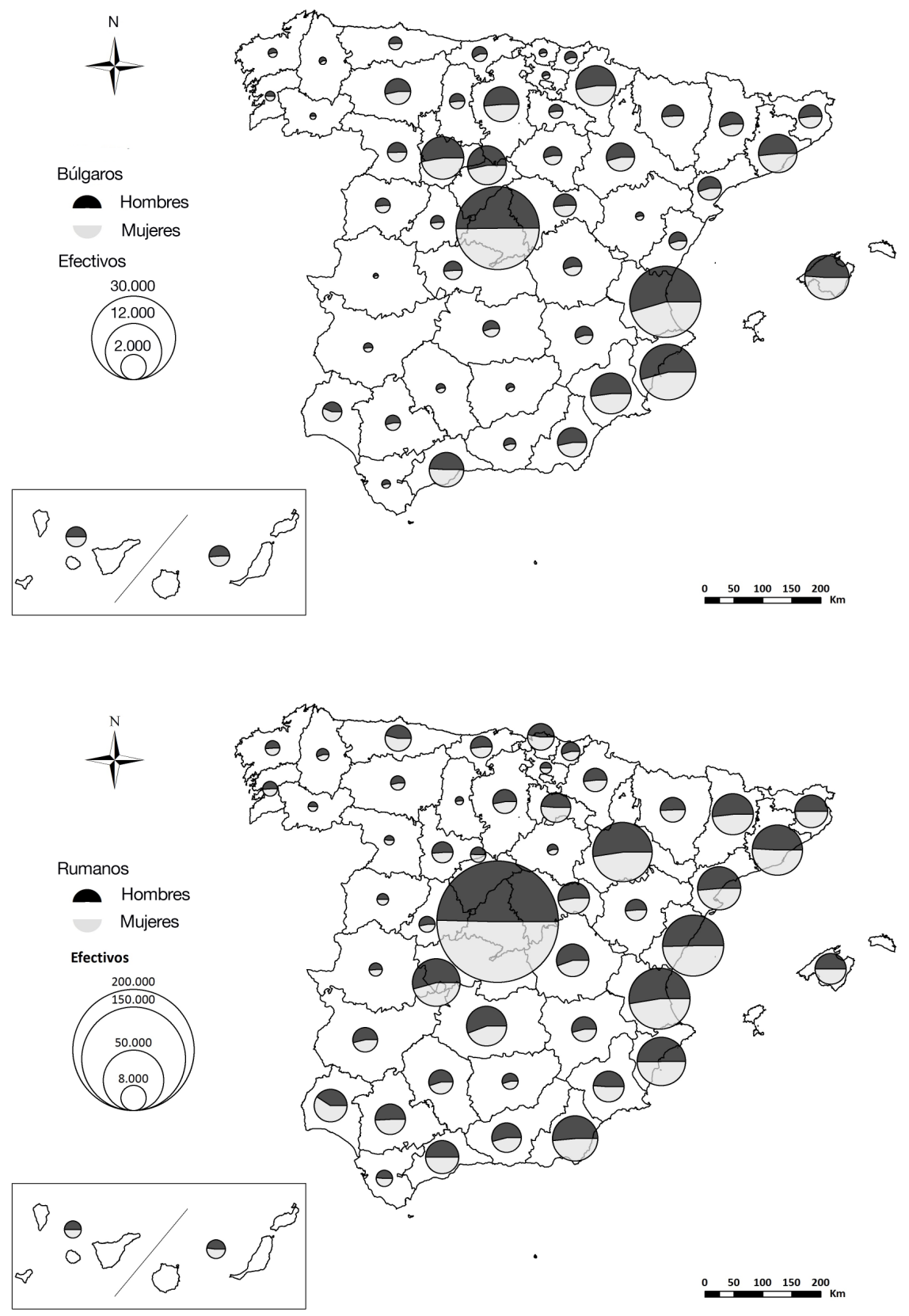

Figura 1. Distribución geográfica de la población nacida en Bulgaria y Rumania (1 de enero de 2013).

Fuente: INE, Padrón de Habitantes. Diseño gráfico de Catherine Andrés Langa. 


\section{Objetivo: el estudio de la movilidad en tiempos de crisis}

La Gran Recesión, iniciada a finales de 2007, tiene efectos severos en la economía española y se manifiesta fundamentalmente como crisis de empleo. En seis años se han destruido tres millones y medio de puestos de trabajo y en la actualidad el número de personas que quieren trabajar y no encuentran dónde hacerlo asciende a 5,9 millones, lo que equivale al 25,9\% de la población activa (primer trimestre de 2014), frente al 9\% de 2007. Las cifras del desempleo alcanzan niveles insoportables sobre todo entre la población inmigrada, cuya tasa de paro $(36,6 \%)$ es doce puntos más elevada que la de la población autóctona $(24,3 \%)$. Los europeos del Este se encuentran entre los más afectados (European Commission, 2011 y 2012). El desempleo se ceba sobre todo en los varones debido a su acusada dependencia del sector de la construcción. En cambio, las mujeres con mayor presencia en los servicios han acusado menos el impacto de la crisis (Muñoz, 2012).

La recesión ha truncado las expectativas de los inmigrantes que se han quedado sin trabajo y de sus familias que han visto cómo sus ingresos disminuyen de forma drástica. La situación de los inmigrantes rumanos y búlgaros, como la de otros colectivos, depende estrechamente del estado del mercado de trabajo, por lo que en las actuales circunstancias cabría esperar un aumento de los flujos de salida y una contracción de los de entrada. La reducción del número de residentes en España (véase la Tabla 1) corrobora el cambio de ciclo migratorio coincidiendo con el agravamiento de la crisis, «la crisis dentro de la crisis» (Oliver, 2013: 30).

España, como otros países de inmigración afectados por la recesión, está interesada en reducir el número de inmigrantes en su territorio y para ello ha adoptado medidas que tratan de frenar las entradas y fomentar las salidas. Así, el Gobierno español impuso una moratoria de dos años a la libre circulación de trabajadores rumanos y búlgaros, entre el 1 de enero de 2007 y el 31 de diciembre de 2008, y a los rumanos, desde el 22 de julio de 2011 hasta el 31 de diciembre de 2013. Unos y otros no pueden acogerse a los programas de retorno aprobados por España en 2008 (retorno voluntario) y 2010 (retorno productivo) por tratarse de ciudadanos comunitarios, pero los gobiernos español y rumano firmaron, en mayo de 2009 , un convenio para facilitar el retorno de trabajadores en paro o en riesgo de perder el empleo que, como los programas dirigidos a inmigrantes de países terceros, ha tenido escaso éxito (Marcu, 2013).

Como ha ocurrido en otros contextos temporales y espaciales (Moen y Wethington, 1992; Sirkeci et al., 2012), frente a la crisis y la incertidumbre, los migrantes despliegan diversas estrategias (aceptan peores condiciones de trabajo, reducen gastos en consumo y vivienda, etc.) con las que tratan de reducir la brecha entre las necesidades familiares y los recursos disponibles. Una de las estrategias consiste en la movilidad geográfica en busca de oportunidades que les permitan mejorar su situación (Massey, 1985; Vertovec, 2007). Las redes sociales tienen un papel clave en la gestión de la movilidad (Hannam, Sheller y Urry, 2006). Son ellas las que sustentan los flujos y los dirigen hacia determina- 
dos lugares (Portes y Böröcz, 1989; Gurak y Caces, 1992). Los migrantes saben cómo desplazarse y adónde dirigirse gracias a la información y los recursos aportados por familiares y conocidos, y aprovechan la presencia de miembros de su red en diferentes lugares. Parafraseando a Emmanuel Ma Mung (1999), la amplitud territorial que han alcanzado los migrantes rumanos y búlgaros, tanto en España como en otros países europeos, se convierte en un recurso.

El estudio de la movilidad debe contemplar tanto la migración interna, entre diferentes regiones del país de destino, como la internacional. Los europeos del Este han exhibido una elevada movilidad interna desde los primeros momentos de su llegada a España, superior a la de otros colectivos extranjeros (Viruela, 2010; Reher y Silvestre, 2011). Los datos aportados por el Instituto Nacional de Estadística (INE) y las crónicas y noticias que publican los medios de comunicación muestran que con la crisis se ha activado la emigración y que el número de inmigrantes que salen de España aumenta de forma progresiva. El artículo indaga en las pautas de movilidad de los migrantes búlgaros y rumanos durante la grave crisis económica que afecta a uno de los principales países de acogida y trata de contribuir al estudio de la movilidad en tiempos de crisis. La investigación pretende responder a preguntas como: ¿qué personas se desplazan?, ¿qué patrones siguen en la movilidad interior? o ¿adónde van los que salen de España?

\section{Metodología}

El artículo combina la metodología cualitativa y la cuantitativa. Para el análisis de la movilidad geográfica interior se han aprovechado los datos recopilados por el Instituto Nacional de Estadística (INE) y el Ministerio de Empleo y Seguridad Social (MESS). El INE elabora la Estadística de Variaciones Residenciales (EVR), que registra los desplazamientos que implican cambio de municipio de residencia (incluidos los que se realizan entre municipios de una misma provincia) y que, en términos generales, suelen afectar a todo el núcleo familiar. La EVR se elabora a partir de las altas y las bajas recogidas en el Padrón de habitantes, un trámite administrativo que interesa a quienes cambian de lugar de residencia porque es un requisito indispensable para acceder a los servicios básicos: escolarización, asistencia sanitaria, etc. Se considera la fuente más importante para cuantificar los flujos migratorios entre los más de 8.000 municipios que hay en España. Por su parte, la base de datos del Servicio Público de Empleo Estatal del MESS registra los contratos ocupados según la nacionalidad y el sexo de los trabajadores, incluyendo los que se realizan en provincias distintas a la de residencia, con desplazamientos normalmente de corta duración, repetidos o cíclicos, que no van acompañados de cambio de domicilio permanente o principal, y que remiten a la movilidad circulatoria en el sentido clásico de Zelinsky (1971). El Observatorio de las Ocupaciones considera que hay movilidad cuando el domicilio del trabajador no coincide con el del centro de trabajo y ha facilitado amablemente las cifras de contratos correspondientes a ciudadanos búlgaros y rumanos, en una matriz similar a 
la que publica para el conjunto de trabajadores con una periodicidad anual, lo que permite analizar la movilidad interprovincial por motivos laborales ${ }^{1}$.

En ambas fuentes de información, la unidad de medida no es el individuo sino la migración y una misma persona puede realizar varios desplazamientos a lo largo de un año. El INE no registra todos los cambios de residencia y algunos se declaran con bastantes meses de retraso, mientras que el MESS solo contabiliza los contratos legales de trabajo y, por tanto, tampoco recoge todos los movimientos ya que en España hay una gran irregularidad en la contratación de la mano de obra. Los datos del Ministerio constituyen una fuente de información complementaria en el estudio de la movilidad geográfica interior, como también lo son los datos de que dispone la Seguridad Social (Pumares et al., 2006), pero los resultados no se pueden comparar con la Estadística de Variaciones Residenciales.

La EVR aporta información de los flujos migratorios con el extranjero. Pero mientras el registro de entradas puede considerarse de buena calidad, el cómputo de salidas es poco fiable (Roig y Recaño, 2012; Domingo y Sabater, 2013), como ocurre en otros países (Lemaitre, 2005). La medición de los flujos de salida presenta mayor dificultad en el caso de los ciudadanos comunitarios que, a diferencia de los migrantes de países terceros, no están obligados a renovar su inscripción como residentes en la oficina municipal de estadística. Según los funcionarios consultados, los rumanos no suelen darse de baja cuando se van, sobre todo cuando parte de la familia sigue viviendo en España. Dicho de otro modo, muchos de los que se han ido constan como residentes en España y ello dificulta el análisis de la emigración. Por otra parte, la inmensa mayoría de los que emigran no indican adónde van.

La metodología cualitativa ha consistido en el análisis de entrevistas en profundidad (entre una hora y una hora y media de duración) a inmigrantes rumanos (41) y búlgaros (23), que se llevaron a cabo en el primer trimestre de 2011 en Valencia, Castellón, Madrid y municipios de su área metropolitana, que se encuentran entre los principales lugares de asentamiento de los inmigrantes de Europa del Este (Figura 1). A estas entrevistas hay que añadir las que se hicieron en los lugares de origen a doce inmigrantes retornados y seis familiares de emigrantes en Tiganesti (Rumania), y otras 10 y 4, respectivamente, en Vidin, Sofia y Varna (Bulgaria) ${ }^{2}$.

Las preguntas incluidas en las entrevistas giran en torno a una gran variedad de temas, que nos aproximan a la vivencia, experiencia y estrategias de los

1. El Observatorio de las Ocupaciones publica los resultados de la movilidad interprovincial de los trabajadores, que se puede consultar en Datos básicos de movilidad. Contratación y movilidad geográfica de los trabajadores en España. <http://www.sepe.es/indiceObservatorio/ buscar.do? tipo $=3$ \&indice $=3$ \&idioma=es $>$. Véase la página 21 del informe editado en 2014 (datos de 2013).

2. Las entrevistas forman parte del trabajo realizado en la ejecución del proyecto I+D+i: Migraciones de la Europa del Este a España en el contexto geopolítico fronterizo: movilidad circulatoria y retorno, financiado por el Ministerio de Ciencia e Innovación (Ref. CSO2010-14870, 2011-2013). 
inmigrantes. Debido a la gran cantidad de información obtenida, se ha optado por el análisis temático, que es un buen ejemplo de la técnica de reducción de datos en la investigación cualitativa (Grbich, 2007). Una vez transcritas las entrevistas, los contenidos se agrupan por grandes bloques temáticos mediante la aplicación del programa de codificación ATLAS.ti. Para este artículo se han aprovechado las cuestiones relativas a la situación en que se encontraban los migrantes en el momento de la entrevista, lo que permite evaluar las estrategias adoptadas por los entrevistados para hacer frente a la grave y persistente crisis que afecta al mercado de trabajo español.

\section{La movilidad geográfica interior}

Pese a la duración y la intensidad que registra la crisis en España, la mayor parte de los europeos del Este han decidido quedarse por diversos motivos. Unos han mejorado social y laboralmente; otros, aunque han perdido el empleo, consideran que están mejor en España que en su país y no encuentran razones suficientes para regresar porque:

En Rumania, si trabajas un mes, apenas puedes vivir una semana... y aquí, si trabajas una semana, puedes vivir un mes. [Por eso] a pesar de la crisis, se está mejor aquí. (Varón, rumano, 24 años)

Bulgaria está peor [que España]. Cuando fui la última vez, estaba todo más caro, los precios de la comida, todo. Una cajera de un supermercado cobra 200 euros y la luz es más cara que aquí. El alquiler [de la vivienda] es más barato, pero la comida está por las nubes, yo no sé cómo vive la gente. (Mujer, búlgara, 41 años)

En general, los migrantes reconocen que están mejor en España que en sus países de origen y para reducir los impactos de la crisis adoptan diversas estrategias, unas en el ámbito reproductivo: ahorran todo lo que pueden, reducen gastos en consumo, vivienda y en el envío de remesas; y otras en el productivo: los miembros de la familia que permanecían inactivos buscan trabajo de forma activa, los que han perdido el empleo intentan encontrar trabajo en otro sector de actividad o en otro lugar.

\subsection{Las variaciones residenciales}

En la etapa de expansión económica, en España se registró un fuerte crecimiento de la movilidad interior de la población inmigrada, en paralelo al aumento de los efectivos (Domingo y Recaño, 2010). Entre 2001 y 2007, las variaciones residenciales de los búlgaros se multiplicaron por diez y las de los rumanos por trece (Tabla 2), cuyas tasas superaban a las de otros colectivos en relación con la mayor propensión a la movilidad de los recién llegados (Recaño, 2002). Según la EVR, los desplazamientos interiores experimentaron una drástica reducción en los dos primeros años de crisis. En efecto, si en 2007 se contabi- 
Tabla 2. Movilidad residencial interior según el lugar de nacimiento (2001-2013)

\begin{tabular}{cccccccc}
\hline & \multicolumn{3}{c}{ Bulgaria } & & \multicolumn{3}{c}{ Rumania } \\
\cline { 2 - 5 } \cline { 6 - 8 } Año & Movimientos & Tasa & TF & & Movimientos & Tasa & TF \\
\hline 2001 & 1.594 & 74,80 & 39,0 & & 5.280 & 103,93 & 36,9 \\
2002 & 6.030 & 144,26 & 40,7 & & 14.972 & 145,08 & 39,7 \\
2003 & 7.852 & 126,90 & 42,7 & & 21.154 & 122,91 & 44,2 \\
2004 & 9.288 & 113,73 & 43,0 & & 27.945 & 107,79 & 45,2 \\
2005 & 10.938 & 112,92 & 42,4 & & 38.050 & 107,28 & 45,9 \\
2006 & 12.343 & 111,74 & 43,4 & & 48.516 & 106,83 & 47,7 \\
2007 & 15.198 & 112,21 & 43,1 & & 68.184 & 112,04 & 46,2 \\
2008 & 10.440 & 67,20 & 45,9 & & 46.927 & 63,92 & 48,0 \\
2009 & 9.243 & 57,14 & 48,7 & & 43.230 & 55,78 & 51,6 \\
2010 & 8.914 & 54,15 & 48,4 & & 44.330 & 55,58 & 52,1 \\
2011 & 9.079 & 54,40 & 47,9 & & 45.504 & 55,35 & 52,7 \\
2012 & 9.081 & 55,32 & 49,8 & & 42.146 & 51,55 & 53,9 \\
2013 & 8.323 & 55,04 & 50,1 & & 39.410 & 51,63 & 54.0 \\
\hline
\end{tabular}

Fuente: INE, Estadística de Variaciones Residenciales. La tasa expresa el número de movimientos por cada 1.000 habitantes. Elaboración propia.

TF: porcentaje de mujeres.

lizaron 112 cambios de municipio de residencia por cada 1.000 inmigrantes rumanos y búlgaros, en 2009 tan solo hubo 56-57. Los valores han seguido menguando desde entonces y son inferiores a los de otros grupos (magrebíes, latinoamericanos), sin duda por el mayor impacto de la crisis en los europeos del Este (Gil et al., 2012).

Hombres y mujeres participan casi por igual en la movilidad residencial, con un destacado protagonismo de los que tienen entre 20 y 40 años (Figura 2). El colectivo rumano destaca por la mayor proporción de mujeres $(54 \%$ del total, en 2013) y por su mayor juventud, los adultos-jóvenes suponen el $65 \%$, frente al $52 \%$ de los búlgaros. Tanto en un caso como en otro, hay una presencia relativamente importante de menores de 16 años (alrededor del 10\% del total). La composición por sexos y la presencia de jóvenes apuntan a que estos desplazamientos movilizan a todos los miembros de la familia.

\subsection{La movilidad interprovincial de trabajadores}

La intensidad y el carácter generalizado de la crisis explican la fuerte reducción de los cambios de municipio de residencia. Sin embargo, según el MESS, el número de contratos ocupados por trabajadores de Europa del Este en provincias distintas a la de residencia casi se duplicó entre 2007 y 2011 (Tabla 3). Es decir, un gran número de migrantes rumanos y búlgaros se han puesto en movimiento y se desplazan a otras provincias por motivos de trabajo. De esta forma intentan sacar el máximo provecho de las escasas oportunidades que, en 

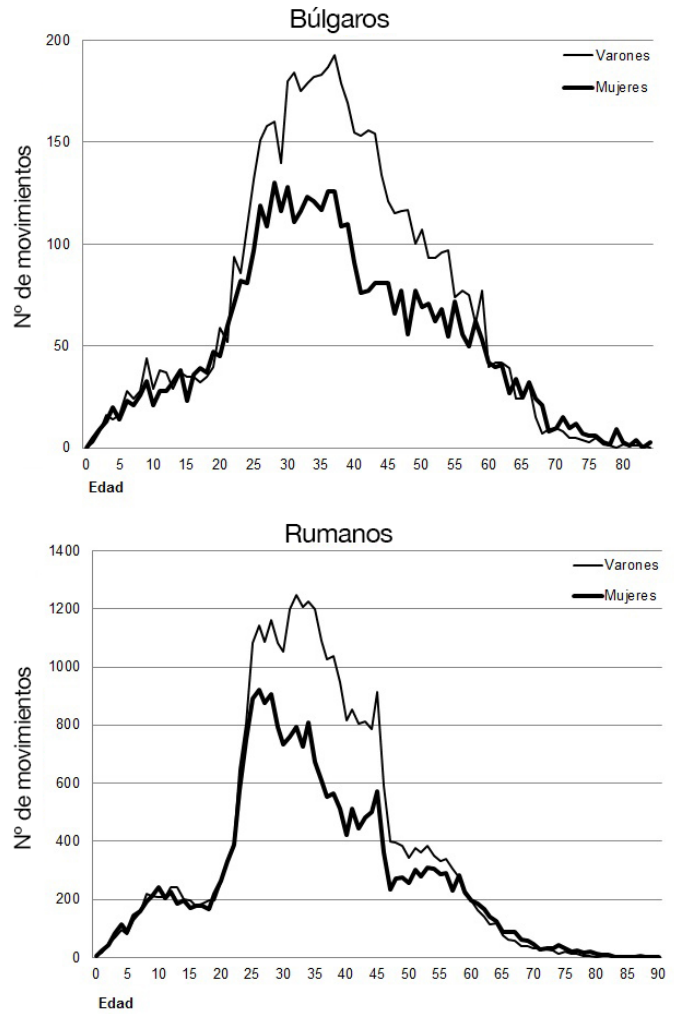

Figura 2. Variaciones residenciales interiores de los inmigrantes nacidos en Bulgaria y Rumania, registradas en 2013, por sexo y edad.

Fuente: INE, Estadística de Variaciones Residenciales (microdatos). Elaboración propia.

Tabla 3. Contratos en provincias distintas a la de residencia (2007-2013)

\begin{tabular}{ccccccccc}
\hline & \multicolumn{3}{c}{ Búlgaros } & & \multicolumn{3}{c}{ Rumanos } \\
\cline { 2 - 5 } \cline { 7 - 8 } Año & $\begin{array}{c}\text { Número } \\
\text { de contratos }\end{array}$ & TM & $\begin{array}{c}\text { \% de } \\
\text { mujeres }\end{array}$ & & $\begin{array}{c}\text { Número } \\
\text { de contratos }\end{array}$ & TM & $\begin{array}{c}\text { \% de } \\
\text { mujeres }\end{array}$ \\
\hline 2007 & 13.066 & 15,6 & 23,5 & & 59.387 & 16,6 & 28,1 \\
2008 & 13.345 & 16,4 & 25,8 & & 64.731 & 17,2 & 29,6 \\
2009 & 15.192 & 17,5 & 28,8 & & 90.264 & 18,9 & 31,3 \\
2010 & 18.885 & 20,9 & 29,3 & & 117.112 & 21,8 & 30,1 \\
2011 & 19.985 & 21,5 & 29,1 & & 117.852 & 22,6 & 31,4 \\
2012 & 18.179 & 18,7 & 33,8 & & 96.828 & 20,3 & 34,8 \\
2013 & 18.893 & 20,7 & 33,4 & & 103.198 & 22,7 & 35,0 \\
\hline
\end{tabular}

Fuente: MESS, Departamento de Estadística del Observatorio de las Ocupaciones. Elaboración propia.

TM: tasa de movilidad como porcentaje de los contratos en provincias distintas a la de residencia con respecto al total de contratos ocupados por cada colectivo. 
las actuales circunstancias, ofrecen los mercados locales de trabajo. Las cifras se han reducido en fecha reciente, pero la proporción de los que trabajan fuera de su provincia se ha mantenido en torno al $20-22 \%$. Los varones son protagonistas en la movilidad laboral y lo más habitual es que se desplacen solos, pero en los últimos años ha aumentado la participación de las mujeres, que ya realizan un tercio o más de estos desplazamientos interprovinciales.

En la migración laboral, el trabajador mantiene la residencia en el lugar de partida y permanece en el lugar de destino un periodo de duración variable, en función de las características de las tareas a realizar y de la disponibilidad y composición de la mano de obra local. La movilidad geográfica de los trabajadores extranjeros que registra el MESS se relaciona fundamentalmente con el sector agrario y, en menor medida, con la hostelería y la construcción. En los últimos años, muchos de los que han perdido el empleo en la construcción, la industria y los servicios buscan refugio en la agricultura y se han convertido en jornaleros itinerantes, participando en diferentes campañas agrícolas. Entre las que atraen un mayor contingente destacan la recolección de aceitunas en Andalucía y Extremadura; ajo y cebolla, además de la vendimia, en Castilla-La Mancha; cítricos en la Comunidad Valenciana y Murcia; fruta en Cataluña y Aragón; el cultivo de la fresa en viveros de Segovia y la recolección de esta fruta en Huelva. Las campañas de recolección requieren mano de obra abundante concentrada en muy poco tiempo. Por este motivo, la estrategia del trabajador consiste en enlazar diferentes campañas en distintas provincias con el fin de mantenerse activo la mayor parte del año. Es habitual que el migrante siga una secuencia tiempo-espacio, condicionada por las oportunidades que brindan los mercados locales de trabajo, que se repite de un año a otro, lo que da a estos desplazamientos el carácter de migración circular.

Entre 2007 y 2011 (el año que registra un mayor número de desplazamientos), el espacio migratorio de los trabajadores búlgaros y rumanos se ha ampliado y a los flujos entre provincias geográficamente próximas, que concentran un mayor número de desplazamientos, se añaden otros que abarcan distancias largas (Figuras 3 y 4). Los itinerarios más importantes tienen lugar entre las principales provincias de asentamiento de los inmigrantes (véase la Figura 1) y muestran el carácter circular de la migración laboral interior. La movilidad geográfica se apoya en una red social amplia y dispersa por un gran número de municipios y provincias. Los trabajadores disponen de información detallada de la época del año y de la duración de las campañas agrícolas por estancias anteriores o porque se la proporcionan personas conocidas que residen en las localidades a las que acuden y que les ofrecen apoyo logístico. En algunas campañas agrícolas, a los rumanos y búlgaros que residen en España se suman compatriotas procedentes de otros países (Francia, Italia o Grecia) e incluso de Rumania y Bulgaria. Una parte de ellos repiten desde hace años las mismas campañas agrícolas y son contratados por los mismos empresarios, una relación que se remonta a los años de la contratación en origen, que dejó de aplicarse a rumanos y búlgaros cuando sus países se incorporaron a la Unión Europea 

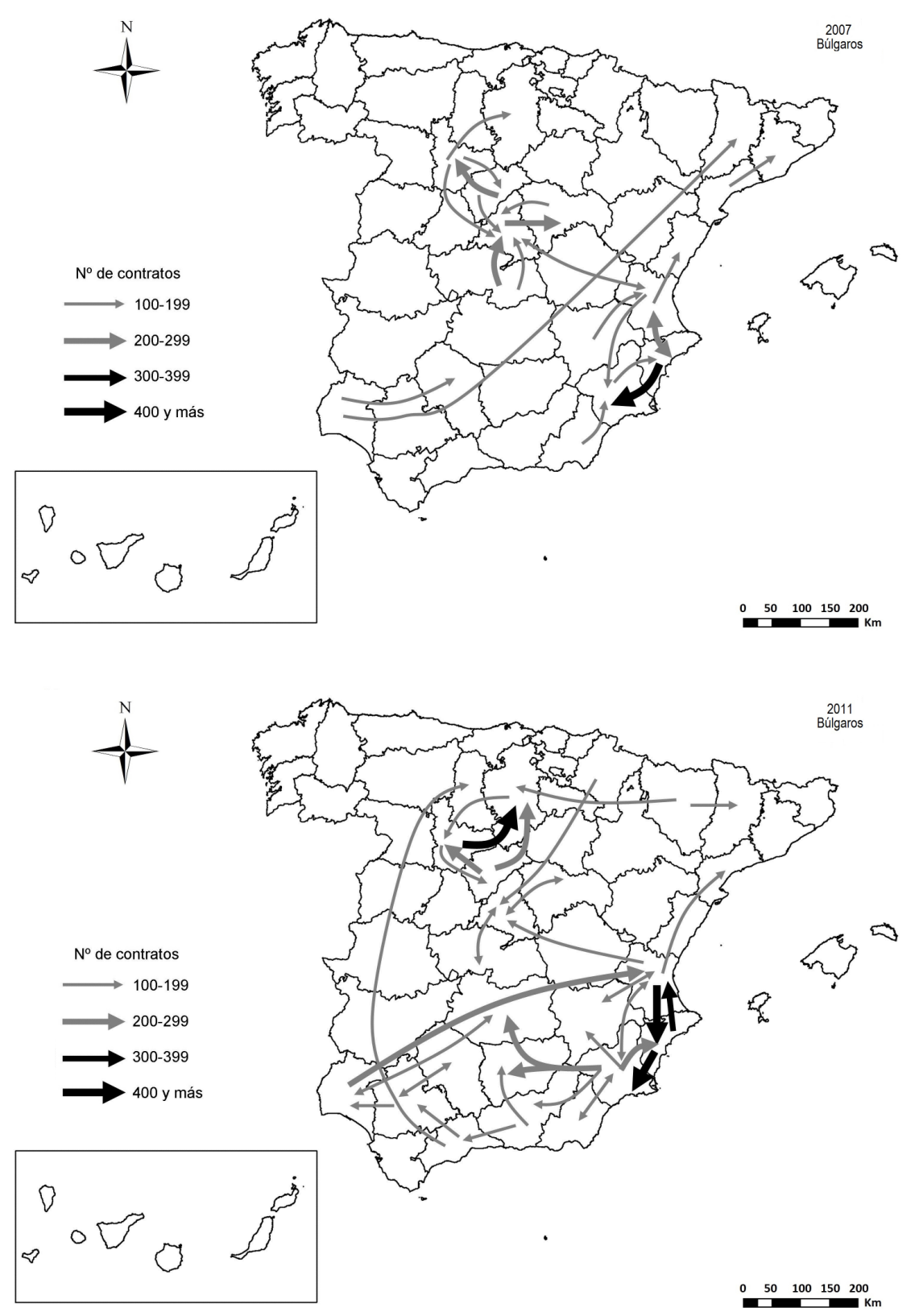

Figura 3. Principales flujos interprovinciales de trabajadores búlgaros, en 2007 y en 2011.

Fuente: MESS, Departamento de Estadística del Observatorio de las Ocupaciones. Elaboración propia. Se representan los flujos que suponen 100 y más contratos en provincias distintas a la de residencia. 


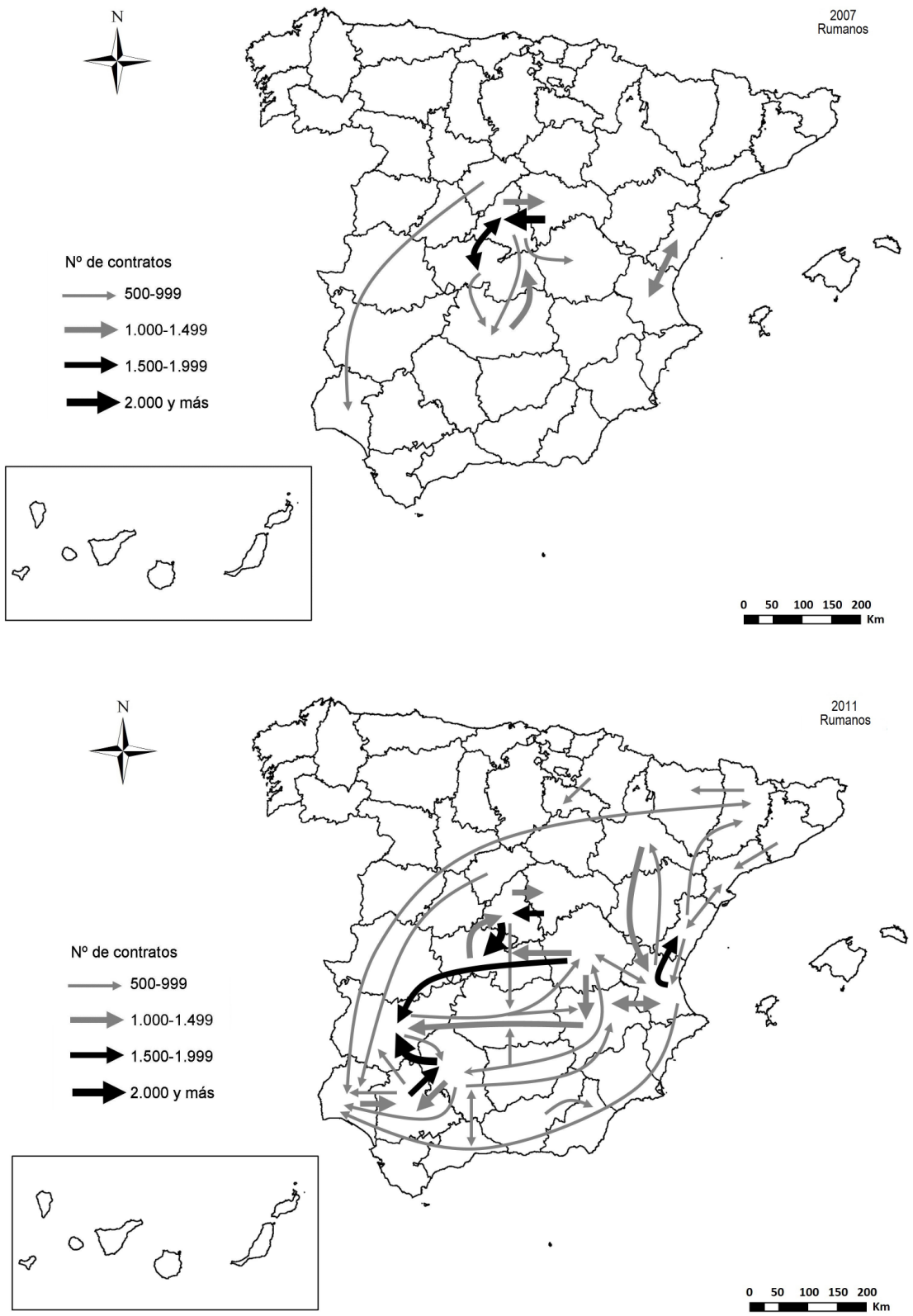

Figura 4. Principales flujos interprovinciales de trabajadores rumanos, en 2007 y en 2011. Fuente: MESS, Departamento de Estadística del Observatorio de las Ocupaciones. Elaboración propia. Se representan los flujos que suponen 500 y más contratos en provincias distintas a la de residencia. 
(Torres et al., 2014). Así, por ejemplo, empresarios de la comarca valenciana de Requena-Utiel dicen que para la vendimia llaman a "su familia rumana».

La reducción de la movilidad laboral en los dos últimos años (véase la Tabla 3) se relaciona con dos motivos. Por una parte, los empresarios del sector agrícola ofrecen empleo a los españoles que han perdido su trabajo en la industria o la construcción, lo que está en consonancia con la preferencia nacional en la contratación (Cebolla y González, 2013). Por otra parte, hay que tener en cuenta el aumento de la emigración.

\section{Estrategias de movilidad internacional}

La crisis económica que sufre España ha tenido un fuerte impacto en las migraciones exteriores. Las entradas se desplomaron en los primeros años, mientras que las salidas han experimentado un progresivo aumento. La emigración ya supera a la inmigración y el stock de residentes en España disminuye (véase la Tabla 1). Según el INE, en los dos últimos años la comunidad búlgara se ha reducido en 25.000 efectivos y la rumana en más de 100.000. Uno de los entrevistados lo expresaba así:

Ya no hay tantos rumanos como en 2007 porque una parte retornó, porque no hay trabajo. Muchos antes tenían trabajo, pero luego perdieron el empleo y la gente retornó o se fue a otros países. (Varón, rumano, 29 años)

Varios miles de migrantes han salido de España en respuesta a la crisis económica. Entre 2008 y 2013, el INE ha certificado más de 41.000 y 200.000 movimientos de salida de búlgaros y rumanos, respectivamente (Tabla 4). Han emigrado jóvenes parejas con hijos pequeños, pero también hay flujos individuales, con mayor proporción de varones entre 25 y 45 años (Figura 5). El

Tabla 4. Flujos de salida de los migrantes nacidos en Bulgaria y Rumania según el destino (2008-2013)

\begin{tabular}{|c|c|c|c|c|c|c|c|c|}
\hline \multirow[b]{2}{*}{ Año } & \multicolumn{4}{|c|}{ Bulgaria } & \multicolumn{4}{|c|}{ Rumania } \\
\hline & Retorno & $\begin{array}{l}\text { Otro destino } \\
\text { conocido }\end{array}$ & $\begin{array}{c}\text { Destino } \\
\text { desconocido }\end{array}$ & Total & Retorno & $\begin{array}{l}\text { Otro destino } \\
\text { conocido }\end{array}$ & $\begin{array}{c}\text { Destino } \\
\text { desconocido }\end{array}$ & Total \\
\hline 2008 & 1.191 & 51 & 1.317 & 2.559 & 5.584 & 155 & 8.060 & 13.799 \\
\hline 2009 & 1.201 & 75 & 3.655 & 4.931 & 5.335 & 140 & 19.955 & 25.430 \\
\hline 2010 & 1.031 & 65 & 6.392 & 7.488 & 4.650 & 189 & 28.401 & 33.240 \\
\hline 2011 & 940 & 81 & 7.745 & 8.766 & 4.801 & 218 & 30.210 & 35.229 \\
\hline 2012 & 1.182 & 93 & 6.236 & 7.511 & 4.987 & 227 & 30.468 & 35.682 \\
\hline 2013 & 1.037 & 72 & 9.229 & 10.338 & 5.205 & 260 & 52.971 & 58.436 \\
\hline Total & 6.582 & 437 & 34.574 & 41.593 & 30.562 & 1.189 & 170.065 & 201.816 \\
\hline$\%(1)$ & 15,8 & 1,1 & 83,1 & 100,0 & 15,1 & 0,6 & 84,3 & 100,0 \\
\hline
\end{tabular}

Fuente: INE, Estadística de Variaciones Residenciales (microdatos). Elaboración propia.

(1) \% sobre el total de cada colectivo. 

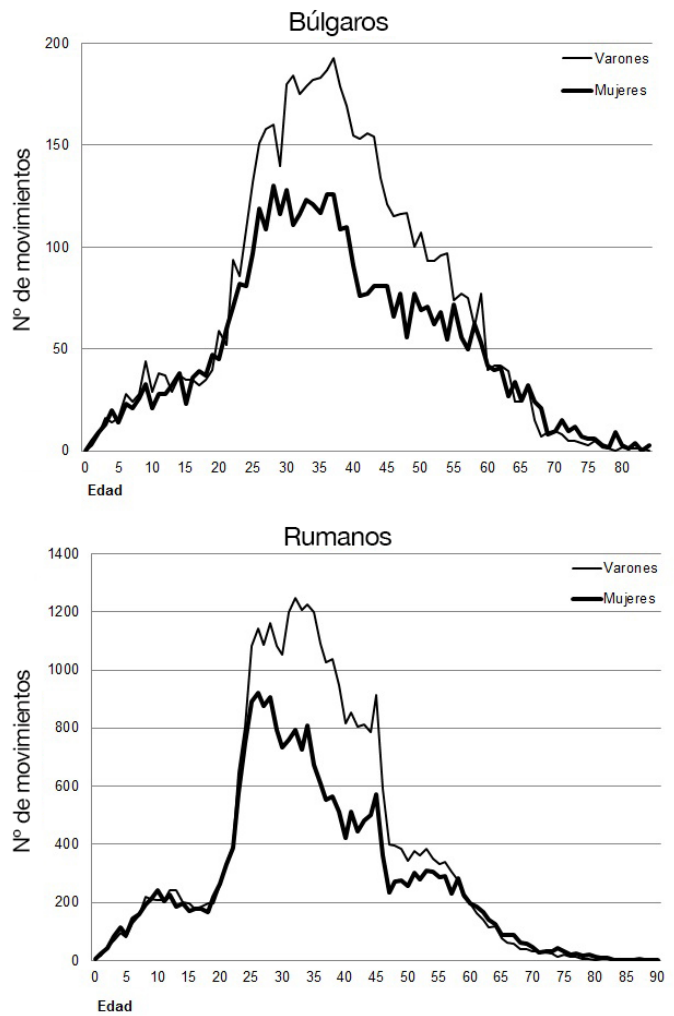

Figura 5. Salidas de inmigrantes nacidos en Bulgaria y Rumania, registradas en 2013, por sexo y edad.

Fuente: INE, Estadística de Variaciones Residenciales (microdatos). Elaboración propia.

desequilibrio entre sexos se relaciona con el fuerte impacto de la crisis en ocupaciones altamente masculinizadas, como la construcción, y con las estrategias familiares. En este sentido, la esposa permanece en España porque tiene más posibilidades de seguir trabajando y por la socialización de los hijos, muchos de los cuales han nacido aquí.

\subsection{Retorno provisional}

La información estadística sobre el destino de los que emigran es muy deficiente ya que se desconoce en el $83-84 \%$ de los casos (Tabla 4). Del resto, sabemos que la mayoría ha retornado a su país de origen, poco más del $15 \%$ de los rumanos y búlgaros que han salido de España en los últimos años. En general, las personas que se han visto más afectadas por la recesión, los jóvenes que tienen cortas trayectorias laborales en España, los parados con dificultades 
de reinserción y que han agotado las prestaciones y los subsidios, los que tienen redes sociales y familiares de menor densidad, consideran que «es hora de volver a casa», a su país, donde pueden subsistir con menos recursos:

Con 100 euros de aquí, allí podemos vivir más tiempo porque en los pueblos la vida es más barata, sobre todo en nuestra región [Neamt, al noroeste de Rumania, una de las regiones más pobres del país]. (Varón, rumano, 24 años)

Unos se refugian en las áreas rurales y trabajan en la pequeña explotación familiar que les proporciona productos de autoconsumo (Bucur, 2012). Otros, ante la ausencia de un sistema salarial sólido, se convierten en trabajadores autónomos invirtiendo sus ahorros en un pequeño negocio (Weber, 2009; Shima, 2010). Pero, con frecuencia, el retorno tiene carácter provisional ya que los que regresan a su país no descartan la posibilidad de volver a salir si no se cumplen sus expectativas, sobre todo cuando tienen familiares, relaciones y contactos en el exterior:

En principio, regresé para quedarme y abrí un negocio, una papelería, en Bucarest. Por ahora, no funciona como me gustaría, pero al menos lo intento. Mi idea es vivir en mi país, aunque lo haré solo si me ayudan las circunstancias y pasa la crisis. De hecho, mi marido y uno de mis hijos se quedaron en España. Yo regresé con otro de mis hijos, y lo bueno es que puedo moverme cuando quiera, y regresar a España si mi negocio no terminara de funcionar. (Mujer, rumana, 52 años)

En general, las inversiones productivas son modestas ya que la mayor parte del dinero se dedica a mejorar el nivel de vida de la familia o se invierte en la construcción de una vivienda (Larionescu, 2012). La situación actual en Bulgaria y Rumania no es la más favorable para el éxito de las iniciativas empresariales de los migrantes: las infraestructuras son deficientes, sobre todo en las áreas rurales, los trámites burocráticos muy lentos y, además, el retornado se enfrenta a la competencia de empresarios occidentales o de compatriotas que no han emigrado y que captan la mayor parte de las ayudas destinadas a la creación de empresas (Weber, 2009).

Los testimonios de las personas entrevistadas confirman los resultados de varios estudios en el sentido de que el retorno no es un fenómeno masivo y que muchos de los que regresan lo hacen por un corto periodo de tiempo (Barbulescu, 2009; Stoiciu et al., 2011; Roman y Goschin, 2012), lo que se debe relacionar con la práctica de la migración circulatoria.

\subsection{Migración circulatoria}

Ante las dificultades para el retorno definitivo, rumanos y búlgaros prefieren y practican la migración circulatoria, que consiste en frecuentes idas y venidas entre el lugar de origen y el de destino. El estatus comunitario permite la libre circulación, que los entrevistados valoran de forma positiva, pese a las restric- 
ciones de acceso al mercado de trabajo impuestas por el Gobierno español. La ciudadanía europea es un "pasaporte» que da derecho a la movilidad, pero los desplazamientos responden fundamentalmente a la difícil situación económico-laboral, que impide a los migrantes asentarse en un único lugar:

Me encantaría tener solo un lugar, pero mi situación económica no me lo permite, tiene que ver con esto, porque si tuviera un trabajo bueno aquí, no volvería a buscarlo también allí. (Mujer, rumana, 43 años)

Las idas y venidas entre el lugar de origen y el de destino son relativamente frecuentes y se aprovechan para explorar las escasas oportunidades que brindan los mercados de trabajo en el lugar de origen y en el de destino, para realizar trabajos temporales, hacer un seguimiento del proceso de construcción de la vivienda, en la que han invertido la mayor parte de los ahorros, o para cuidar de los padres ancianos y de los hijos, como también han destacado otros autores (Sintès, 2007; Stoiciu et al., 2011; Pérez, 2012).

Los migrantes de Europa del Este, tienen una larga experiencia en la movilidad, que registró notoriedad tras el colapso del bloque comunista (Morokvasic, 1999; Wihtol, 2001) y, en fecha reciente, con la exención de visado y la ampliación de la Unión Europea. Salir y regresar ha sido desde entonces mucho más fácil (Favell, 2008). Los testimonios de las personas entrevistadas sugieren que en los últimos años se ha producido un notable aumento de los desplazamientos entre el lugar de origen y el de destino, que no es posible cuantificar y que obedece fundamentalmente a las dificultades económico-laborales del momento. Desplazamientos que, por otra parte, se han visto favorecidos por la proximidad geográfica, la facilidad de movimientos en el espacio comunitario, y el desarrollo y abaratamiento de los transportes.

\subsection{Emigración a un tercer país}

La emigración a un tercer país es minoritaria pero va en aumento («otro destino conocido» en la Tabla 4). Los búlgaros y rumanos prefieren otro país de Europa occidental como lugar de destino: Alemania, Italia, Reino Unido, etc. (Tabla 5). Una elección que está condicionada por el conocimiento directo que tiene el migrante por estancias anteriores a su llegada a España y por la presencia de familiares y amigos.

Familias enteras han salido de España buscando oportunidades en otro país, pero es más habitual, sobre todo entre los rumanos, que emigre el cabeza de familia en situación de desempleo, mientras que el cónyuge permanece en España con los hijos. Los responsables de Cáritas de Castellón han observado que «muchos padres rumanos se van a otros países europeos y desde allí mantienen a la familia que ha quedado aquí, para que los menores no pierdan la escuela, la atención sanitaria, etc.» La separación se considera la solución más adecuada para optimizar los recursos económicos y para la socialización de los hijos, y es una opción que se ve facilitada por la posibilidad que tiene el 
Tabla 5. Principales países de destino de los migrantes búlgaros y rumanos (2008-2013)

\begin{tabular}{|c|c|c|c|c|c|c|c|}
\hline \multirow[b]{3}{*}{ País de destino } & \multicolumn{3}{|l|}{ Bulgaria } & \multicolumn{4}{|c|}{ Rumania } \\
\hline & \multicolumn{3}{|c|}{$\mathrm{N}^{0}$ de movimientos de salida } & \multirow[b]{2}{*}{ País de destino } & \multicolumn{3}{|c|}{$\mathrm{N}^{\circ}$ de movimientos de salida } \\
\hline & Varones & Mujeres & Total & & Varones & Mujeres & Total \\
\hline Alemania & 34 & 39 & 73 & Alemania & 122 & 116 & 238 \\
\hline Reino Unido & 23 & 30 & 53 & Italia & 108 & 121 & 229 \\
\hline Rumania & 31 & 22 & 53 & Francia & 73 & 46 & 119 \\
\hline Francia & 17 & 15 & 32 & Reino Unido & 57 & 40 & 97 \\
\hline Italia & 12 & 17 & 29 & Portugal & 44 & 31 & 75 \\
\hline Grecia & 7 & 18 & 25 & Bélgica & 30 & 33 & 63 \\
\hline Portugal & 13 & 12 & 25 & Marruecos & 24 & 12 & 36 \\
\hline EE UU & 9 & 10 & 19 & Austria & 17 & 17 & 34 \\
\hline Bélgica & 10 & 7 & 17 & Moldavia & 17 & 13 & 30 \\
\hline Holanda & 7 & 6 & 13 & Bulgaria & 10 & 12 & 22 \\
\hline Otros países & 49 & 49 & 98 & Otros países & 118 & 128 & 246 \\
\hline Total & 212 & 225 & 437 & TOTAL & 620 & 569 & 1.189 \\
\hline
\end{tabular}

Fuente: INE, Estadística de Variaciones Residenciales (microdatos). Elaboración propia.

migrante de seguir cobrando el subsidio de desempleo mientras busca trabajo en otro país de la Unión ${ }^{3}$.

Los jóvenes con estudios universitarios muestran una gran propensión a emigrar a un tercer país ya que en el lugar de origen los salarios son muy bajos y en España, en las actuales circunstancias, tienen serias dificultades para acceder al mercado de trabajo cualificado, como les ocurre a miles de jóvenes españoles.

\section{Conclusión}

La drástica reducción del empleo (3,5 millones en un lustro) y el creciente deterioro del mercado de trabajo constituyen el reverso del modelo de crecimiento previo. En los primeros años del siglo Xxi llegaron a España millones de inmigrantes, muchos de ellos procedentes de Europa del Este, atraídos por la facilidad de encontrar empleo. En la actualidad, en España hay menos trabajo, los ingresos de las familias disminuyen y la pobreza y los procesos de exclusión social aumentan (Laparra y Pérez, 2012). Los inmigrantes padecen más el acelerado deterioro de las condiciones de vida. Para reducir los impactos de la crisis, los recién llegados adoptan diversas estrategias, entre las que destaca la movilidad geográfica, tanto la que tiene lugar entre diferentes localidades y provincias españolas como la migración internacional.

Las estadísticas utilizadas ofrecen diferentes realidades de la movilidad geográfica interior. Por una parte, las variaciones residenciales (INE) se han reduci-

3. Para más información, véase la conferencia pronunciada por László Andor en la Universidad de Bristol, el 10 de febrero de 2014: <http://europa.eu/rapid/press-release_SPEECH-14-115_en.htm> [consulta: 28 de febrero de 2014]. 
do de forma considerable durante la crisis, sobre todo en los primeros años. Por otra parte, ha aumentado la contratación de trabajadores en provincias distintas a la de residencia (Observatorio de las Ocupaciones, MESS). Conviene recordar que este tipo de desplazamiento no supone cambio de residencia principal y que por ello no queda constancia en la Estadística de Variaciones Residenciales. La movilidad laboral interprovincial, protagonizada por varones, está estrechamente relacionada (aunque no solo) con la concatenación de diferentes campañas agrícolas en distintas provincias y regiones españolas. Predominan los desplazamientos cortos, entre provincias vecinas, pero con la crisis el campo migratorio se amplia y los trabajadores incorporan otros espacios más alejados e intentan mantenerse activos durante más tiempo. La reciente reducción de la contratación de inmigrantes búlgaros y rumanos en provincias distintas a la de residencia puede estar relacionada con la agudización de la crisis, la competencia de trabajadores españoles y la preferencia nacional en la contratación de mano de obra, y con el aumento de la migración internacional.

La crisis ha tenido un fuerte impacto en los flujos exteriores: disminuyen las entradas y aumentan las salidas, sobre todo en los dos últimos años, aunque los que se van son una minoría. La emigración aumenta como respuesta al creciente desempleo y al aumento de la precariedad. Se han ido familias completas, pero la emigración está protagonizada por varones adultos-jóvenes. El cabeza de familia, más afectado por el desempleo, busca oportunidades en otro lugar, mientras que el resto de la familia permanece en España, donde la esposa tiene más posibilidades de seguir trabajando y para no modificar el entorno de los hijos. La información estadística y los resultados de las entrevistas revelan que la migración exterior adopta diversas formas: retorno, migración circular entre el país de origen y el de destino, y emigración a un tercer país.

El país de origen es el principal destino de los migrantes que salen de España. El retorno definitivo o prolongado es un fenómeno marginal, pero podría aumentar si la situación laboral en España no experimenta un cambio sustancial y, muy especialmente, en la medida en que las mujeres encuentren dificultades para conservar el empleo o tal como vayan desapareciendo las prestaciones por desempleo. No obstante, hay que tener en cuenta que la decisión de regresar depende fundamentalmente de las circunstancias socioeconómicas en el país de origen, donde las posibilidades de obtener un trabajo que satisfaga los objetivos de progreso y remuneración de los migrantes son más bien escasas. Los europeos del Este contemplan el retorno desde la óptica de la movilidad ya que muchos de los que regresan, en realidad, circulan, van y vienen o no descartan futuras migraciones si no se cumplen sus planes y expectativas. Los testimonios de las personas entrevistadas sugieren un notable aumento de la migración circulatoria entre el lugar de origen y el de destino, que no es posible cuantificar. Rumanos y búlgaros tienen una larga experiencia en la movilidad, pero es en los últimos años cuando las idas y venidas atañen a un mayor número de personas, de las que muchas vivían antes en la inmovilidad. La emigración a un tercer país es una opción minoritaria pero va aumentando, con una clara preferencia por los países de Europa occidental (Alemania, Italia, 
Reino Unido, etc.). La elección de estos destinos se relaciona con la proximidad geográfica, el establecimiento de cadenas y de redes migratorias anteriores al boom de la inmigración en España y con las posibilidades de movilidad que ofrece el espacio comunitario a sus ciudadanos.

En definitiva, las estrategias de movilidad, en sus diferentes modalidades, ponen de relieve las dificultades que, en las actuales circunstancias, tienen los migrantes para asentarse en un único lugar. La experiencia acumulada y la información que proporcionan familiares o amigos influyen en la elección de los destinos, de los trayectos a seguir o del momento en que se produce el desplazamiento. La amplitud geográfica que han logrado los rumanos y los búlgaros en España y en otros países europeos se convierte en un recurso de gran valor que facilita la movilidad, con la que los migrantes pretenden aprovechar las escasas oportunidades que, en el momento actual, ofrecen los mercados locales de trabajo.

\section{Referencias bibliográficas}

Barbulescu, Roxana (2009). The Economic Crisis and its Effects for Intra-European Movement: Mobility Patterns and State Responses: The Case of Romanians in Spain. Oxford: University of Oxford: Centre on Migration, Policy and Society. <http://www.compas.ox.ac.uk/fileadmin/files/Events/Annual_conferences/ conference_2009/C_Barbulescu_intra-european\%20migration_01.pdf> [consulta: 6 de marzo de 2014].

Bucur, Ramona (2012). Réseaux migratoires roumains en Espagne. Stratégies et territoires de vie à Castellón de la Plana (Comunidad Valenciana). Thèse de Doctorat. Directeurs Joan Serafí Bernat et Jean Baptiste Humeau. Angers: Université d'Angers.

САС̆ÓN, Lorenzo (2009). La España inmigrante: marco discriminatorio, mercado de trabajo y politicas de integración. Barcelona: Antrophos.

Cebolla, Héctor y GonZÁlez, Amparo (coord.) (2013). Inmigración, ¿integración sin modelo? Madrid: Alianza Editorial.

Colectivo IoÉ (2012). Impactos de la crisis sobre la población inmigrante. <http:// www.colectivoioe.org/uploads/0bae582aa3b0842a9eaf50cde16f4f97d 9527bcb. pdf> [consulta: 22 de enero de 2014].

Colectivo Ió́ y Fernández, Mercedes (2010). Encuesta Nacional de Inmigrantes 2007: el mercado de trabajo y las redes sociales de los inmigrantes. Madrid: Observatorio Permanente de la Inmigración.

Domingo, Andreu y Recaño, Joaquín (2010). «La inflexión en el ciclo migratorio internacional en España: impacto y consecuencias demográficas». En: AJA, Eliseo; Arango, Joaquín y Oliver, Josep (dir.). Anuario de la inmigración en España 2009. Barcelona: CIDOB edicions, 182-207.

Domingo, Andreu y Sabater, Albert (2013). «Crisis económica y emigración: la perspectiva demográfica». En: AJA, Eliseo, Arango, Joaquín y Oliver, Josep (dir.). Anuario de la inmigración en España 2012. Barcelona: CIDOB edicions, 60-87.

European Commission (2011). Report from the Commission to the Council on the Functioning of the Transitional Arrangements on Free Movement of Workers from Bulgaria and Romania. Bruselas, COM (2011) 729 final. <http://ec.europa.eu/ social/BlobServlet?docId=7204\&langId=en> [consulta: 12 de enero de 2014]. 
- (2012). Commission Decision of 20 December 2012 authorising Spain to extend the temporary suspension of the application of Articles 1 to 6 of Regulation (EU) No. 492/2011 of the European Parliament and of the Council on freedom of movement for workers within the Union with regard to Romanian workers, Official Journal of the European Union, 22 de diciembre de 2012. <http://eur-lex.europa.eu/JOHtml. do?uri=OJ:L:2012:356:SOM:EN:HTML> [consulta: 12 de enero de 2014].

Favell, Adrian (2008). «The new Face of East-West Migration in Europe». Journal of Ethnic Migration Studies, 34 (5), 701-716. <http://dx.doi.org/10.1080/13691830802105947>

Gil, Fernando, Bayona, Jordi y Vono, Daniela (2012). «Las migraciones internas de los latinoamericanos en España: del boom a la crisis económica». Papeles de Población, 18 (71), 1-42. <http://www.redalyc.org/articulo.oa?id=11223031002> [consulta: 22 de junio de 2014].

Gómez, Silvia y Molina, José Luis (2010). «Les nouvelles migrations dans l'Europe: chaînes migratoires, établissement et réseaux sociaux des Bulgares en Espagne et en Catalogne». Balkanologie, 12 (2). <http://balkanologie.revues.org/2211> [consulta: 4 de abril de 2014].

Grbich, Carol (2007). Qualitative Data Analysis: An introduction. Londres: Sage.

GuraK, Douglas y CACES, Fe (1992). "Migration networks and the shaping of migration systems”. En: Kritz, Mary M.; LiM, Lin L. y ZLOTNiK, Hania (eds.). International Migration Systems: A Global Approach. Oxford: Clarendon Press, 150-176.

Hannan, Kevin, Sheller, Mimi y Urry, John (2006). «Editorial: Mobilities, Immobilities and Moorings». Mobilities, 1 (1), 1-22. <http://dx.doi.org/10.1080/17450100500489189>

Laparra, Miguel y Pérez, Begoña (2012). Crisis y fractura social en Europa. Causas y efectos en España. Barcelona: Obra Social La Caixa.

LARIONESCU, Andrea Letitia (2012). Locuintele migrantilor în tara de origine. Un studiu decaz despre transformarea locuintei rurale sub impactul migratiei internationale. Comuna Marginea, jud. Suceava. Thèse pour le Doctorat en cotutelle internationale. Directeur Poliana Stefanescu et Guy Tapie. Burdeos: Université Bordeaux Segalen.

Lemaitre, Georges (2005). «The Comparability of International Migration Statistics: Problems and Prospects». Statistics Brief, 9. <http://www.oecd.org/migration/ mig/36064929.pdf> [consulta: 8 de marzo de 2014].

Ma Mung, Emmanuel (1999). «La dispersion comme ressource». Cultures \& Conflits, 33-34, 89-103. <http://conflits.revues.org/225> [consulta: 17 de diciembre de 2013].

Marcu, Silvia (2013). «La movilidad transfronteriza de rumanos en España en tiempos de crisis». Revista Internacional de Sociología, 71 (1), 115-141. <http://dx.doi.org/10.3989/ris.2012.01.18>

Massey, Douglas S. (1985). «Ethnic Residential Segregation: A Theoretical Synthesis and Empirical Review». Sociology and Social Research, 69, (3), 315-350.

Moen, Phyllis y Wethington, Elaine (1992). «The concept of family adaptive strategies». Annual Review of Sociology, 18, 233-251. <http://dx.doi.org/10.1146/annurev.so.18.080192.001313>

Moreno, Francisco Javier y Bruquetas, María (2011). Inmigración y Estado de bienestar en España. Barcelona: Obra Social La Caixa.

Morokvasic, Mirjana (1999). "La mobilité transnationale comme ressource: le cas des migrants de l'Europe de l'Est». Cultures \& Conflits, 33-34, 105-122. <http:// conflits.revues.org/263?lang=es> [consulta: 22 de diciembre de 2013]. 
Muñoz, Jacobo (2012). «Evolución del empleo y el paro de las mujeres inmigrantes en el mercado de trabajo español. El impacto de la actual crisis económica». Cuadernos de Relaciones Laborales, 30, (1), 115-137.

OeCD (2007). International Migration Outlook 2007. París. OECD. <http://www. oecd.org/centrodemexico/publicaciones/migracion.htm $>$ [consulta: 7 de enero de 2014].

Oliver, Josep (2013). «La inmigración y la doble recesión del mercado de trabajo en España, 2011-12». En: Aja, Eliseo, Arango, Joaquín y Oliver, Josep (dir.). Anuario de la inmigración en España 2012. Barcelona: CIDOB edicions, 28-58.

Pajares, Miguel (2010). Inmigración y mercado de trabajo. Informe 2009. Madrid: Observatorio Permanente de la Inmigración.

Pérez, Antía (2012). «El retorno de los migrantes rumanos: inversión de remesas y ahorros y estrategias de reintegración». En: Aвoussi, Mourad, (coord.). El codesarrollo a debate. Granada: Editorial Comares, 71-85.

Portes, Alejandro y Böröcz, Jozsef (1989). "Contemporary Immigration: Theoretical Perspectives on Its Determinants and Modes of Incorporation». International Migration Review, 3, 606-630.

Pumares, Pablo; García, Arlinda y Asensio, Ángeles (2006). La movilidad laboraly geográfica de la población extranjera en España. Madrid: Observatorio Permanente de la Inmigración.

RecaÑo, Joaquín (2002). «La movilidad geográfica de la población extranjera en España: un fenómeno emergente». Cuadernos de Geografía, 72, 135-156.

Reher, David y Silvestre, Javier (2011). «International Migration Patterns of Foreign-Born Immigrants in Spain. A Study Based on the National Immigrant Survey (ENI-2007)». Revista Internacional de Sociología (RIS), Monographic 1, 167-188.

Roig, Marta y Recaño, Joaquín (2012). «The Impact of the Global Financial Crisis on Migration to and Remittance Flows from Spain». En: Sirkeci, Ibrahim, Cohen, Jeffrey H. y Ratha, Dilip (eds.). Migration and Remittances during the global Financial Crisis and Beyond Washington DC: World Bank, 255-269.

Roman, Monica y Goschin, Zizi (2012). «Romanian Immigrants Worldwide: What makes them Return Home?». Journal of Identity and Migration Studies, 6 (2), 2-17. <http://e-migration.ro/jims/Vol6_No2_2012/Articles/JIMS_Vol6_No2_2012_ pp_2_17_ROMAN_GOSCHIN.pdf> [consulta: 9 de septiembre de 2013].

ŞErban, Monica y Voicu, Bogdan (2010). «Romanian Migrants to Spain: In-or Outside the Migration Networks. A Matter of Time?». Revue d'Études Comparatives Est-Ouest, 41, (4), 97-124. <http://dx.doi.org/10.4074/S0338059910004055>

Shima, Isilda (2009). "Return migration and labour market outcomes of the returnees. Does the return really pay off? The case-study of Romania and Bulgaria». EconPapers. FIW Research Reports, 7, 1-50. <http://econpapers.repec.org/paper/ wsrecbook/2010_3ai_3aii-007.htm> [consulta: 14 de julio de 2013].

Sintès, Pierre (2007). "Les travailleurs balkaniques en Grèce. Migration de travail ou circulation régionale?». L'Espace Géographique, 4, 353-365.

Sirkeci, Ibrahim, Cohen, Jeffrey H. y Ratha, Dilit (2012). Migration and Remittances during the global Financial Crisis and Beyond. Washington DC: World Bank.

Stoiciu, Victoria; Stanculescu, Manuela; Alexe, Iris y Motoc, Luminita (2011). Impactul crizei economice asupra migratiei fortei de munca romanesti. Bucarest: Friedrich Ebert Foundation. <http://www.fes.ro/media/images/publications/Impactul_crizei.pdf> [consulta: 13 de septiembre de 2013]. 
Torres, Teresa; Allepuz, Rafael y Gordo, Mercedes (2014). «La contratación de mano de obra temporal en la agricultura hortofrutícola española». Ager. Revista de Estudios sobre Despoblación y Desarrollo Rural, 16, 7-37.

Torres, Francisco (2011). La inserción de los inmigrantes. Luces y sombras de un proceso. Madrid: Talasa Ediciones, S. L.

Vertovec, Steven (2007). Circular Migration. The Way Forward in Global Policy? Oxford: International Migration Institute, University of Oxford. <http://www. imi.ox.ac.uk/pdfs/wp/wp-04-07.pdf> [consulta: 30 de noviembre de 2013].

Viruela, Rafael (2008). «Población rumana y búlgara en España: evolución, distribución geográfica y flujos migratorios». Cuadernos de Geografia, 84, 169-194.

- (2010). «Movilidad geográfica de los rumanos (estructura territorial de las migraciones interiores en España)». Empiria. Revista de Metodología de Ciencias Sociales, 19, 157-181.

- (2011). "The Romanian Migrants in Spain. An Exceptional Migratory Flow». International Review of Social Research 1, 31-59. <http://www.irsr.eu/archive.html> [consulta: 4 de febrero de 2014].

Weber, Serge (2009). "Les mobilités induites par les migrations: émergence d'un camp circulatoire transnational». Méditerranée, 116. <http://mediterranee.revues. org/3790> [consulta : 9 de octubre de 2013].

Wintol, Catherine. (2001). «Un essai de typologie des nouvelles mobilités». Hommes \& Migrations, 1.233, 5-12.

WORLD BANK (2012). Bilateral remittance estimates using migrant stocks, destination country incomes, and source country incomes. <http://go.worldbank.org/ JITC7NYTT0> [consulta: 5 de enero de 2014].

ZeLINsky, Wilbur (1971). «The Hypothesis of the Mobility Transition». Geographical Review, 61 (2), 219-229.

<http://dx.doi.org/10.2307/213996> 